\title{
Primary Hyperaldosteronism: Screening, Diagnosis, and Management for the Clinician
}

\author{
Eugene T. Van, DO ${ }^{1}$, Winnie Nhan, DO ${ }^{2 *}$, Kristyn Perry, DO ${ }^{3}$, Azadeh Brumand, DO ${ }^{4}$ \\ Serena Shen, DO ${ }^{5}$, Kelvin L. Tran, DO ${ }^{6}$, Quang T. Nguyen, DO, FACP, FACE, FTOS \\ 1,2,3,4,6 Department of Internal Medicine, Valley Hospital Medical Center, Las Vegas, NV. \\ ${ }^{5}$ Department of Family Medicine, Valley Hospital Medical Center, Las Vegas, NV. \\ ${ }^{7}$ Medical Director, Las Vegas Endocrinology, Clinical Associate Professor, Clinical Education, AZCOM, and \\ Adjunct Associate Professor of Endocrinology, Touro University Nevada. \\ *Corresponding Author: Winnie Nhan, DO, Department of Internal Medicine, Valley Hospital Medical Center, Las \\ Vegas, NV, USA.

\section{Abstract} \\ Primary hyperaldosteronism (PA) is the most common secondary, non-iatrogenic cause of hypertension. \\ This condition is associated with significant risk of morbidity and mortality, yet it is often unrecognized \\ and undiagnosed in the primary care setting. Screening with the aldosterone to renin ratio (ARR) should be \\ considered in patients with resistant hypertension, defined as blood pressure $>140 / 90 \mathrm{mmHg}$ despite the use of \\ three different classes of antihypertensive medications, including a diuretic. The goal of this review is to inform \\ the primary care clinician of the current guideline recommendations for screening, confirming, subtyping, and \\ treating primary hyperaldosteronism.
}

\section{INTRODUCTION}

Hypertension affects up to 75 million adults in America, with an estimated cost of $\$ 46$ billion in annual health care utilization, prescription medications, and missed work days (11). Patients with hypertension are at an increased risk for heart disease and stroke, which are the first and third leading cause of death in the United States, respectively (4). Although primary or essential Table 1. Overview of secondary causes of hypertension hypertension is frequently recognized and treated in the primary care setting, secondary hypertension is often overlooked and remains untreated. The most common cause of secondary hypertension is hyperaldosteronism and evidence suggests it is associated with higher risk of cardiac disease, renal failure as well as cerebrovascular accidents compared to essential hypertension alone (Table 1) (12).

\begin{tabular}{|l|l|}
\hline Secondary causes of Hypertension & $\begin{array}{l}\text { OCP, Pseudoephedrine, NSAID, TCA, SSRI, Glucocorticoid, } \\
\text { Decongestants Weigh loss agents, Methylphenidate, } \\
\text { Methamphetamine, Cocaine. }\end{array}$ \\
\hline Primary hyperaldosteronism & $\begin{array}{l}\text { Most common etiology is bilateral idiopathic adrenal hyperplasia, } \\
\text { followed by aldosterone-producing adenoma, and primary } \\
\text { adrenal hyperplasia. Rare causes include aldosterone-producing } \\
\text { adrenocortical carcinoma, ectopic aldosterone-producing } \\
\text { adenomas, and familial hyperaldosteronism. }\end{array}$ \\
\hline Secondary hyperaldosteronism & $\begin{array}{l}\text { Exogenousmineralocorticoiduse,nephroticsyndrome,pregnancy, } \\
\text { renovascular disease fibromuscular dysplasia in children and } \\
\text { atherosclerosis including renal artery stenosis in adults), renin- } \\
\text { secreting tumor. }\end{array}$ \\
\hline
\end{tabular}


Primary Hyperaldosteronism: Screening, Diagnosis, and Management for the Clinician

\begin{tabular}{|c|c|}
\hline Obstructive sleep apnea (OSA) & $\begin{array}{l}\text { Blood pressure fluctuates due to a significant increase in } \\
\text { sympathetic activity during sleep. Patients with OSA often retain } \\
\text { sodium and fail to respond to anti-hypertensive drug therapy (10). }\end{array}$ \\
\hline Pheochromocytoma & $\begin{array}{l}\text { Rare but fatal, caused by a functional adrenal mass. Patients } \\
\text { present with paroxysmal hypertension. }\end{array}$ \\
\hline Cushing's syndrome & $\begin{array}{l}\text { Exogenous steroid use, adrenal masses, adrenal hyperplasia, and } \\
\text { ectopic sources abnormally increase the level of glucocorticoid. }\end{array}$ \\
\hline Coarctation of the aorta & $\begin{array}{l}\text { Congenital disease with unknown pathophysiology. Patients } \\
\text { present with hypertension in upper extremities and low or } \\
\text { unobtainable arterial blood pressure in the lower extremities. }\end{array}$ \\
\hline Hyperthyroidism & $\begin{array}{l}\text { Hyperthyroidism has been shown to increase systolic ambulatory } \\
\text { blood pressure compared to euthyroid patients (8). Patients have } \\
\text { increased heart rate, decreased systemic vascular resistance, and } \\
\text { increased cardiac output and stroke volume. }\end{array}$ \\
\hline Hypothyroidism & $\begin{array}{l}\text { Positive correlation between serum TSH levels and blood pressure } \\
\text { specifically increased diastolic pressure and causing an increase } \\
\text { systemic vascular resistance (22). }\end{array}$ \\
\hline Congenital adrenal hyperplasia (CAH) & $\begin{array}{l}\text { 21-hydroxylase deficiency is the most common and causes } \\
\text { impaired synthesis of glucocorticoid and mineralocorticoid } \\
\text { leading to downstream androgen overproduction (16). } \\
17 \alpha \text {-hydroxylase deficiency is rare, but causes increase in } \\
\text { mineralocorticoids from excess conversion of upstream } \\
\text { precursors (2). }\end{array}$ \\
\hline Liddle's Syndrome & $\begin{array}{l}\text { An autosomal dominant condition characterized by over-activity } \\
\text { of the epithelial sodium channel (ENaCs) leading to the triad of } \\
\text { hypertension, hypokalemia, and metabolic alkalosis. }\end{array}$ \\
\hline Primary hyperparathyroidism (PHPT) & $\begin{array}{l}\text { PHPT is associated with hypertension, studies show a correlation } \\
\text { between hypercalcemia and hypertension (9). However, the } \\
\text { etiology is unknown in multiple endocrine neoplasia as treatment } \\
\text { does not improve hypertension (23). }\end{array}$ \\
\hline Acromegaly & $\begin{array}{l}\text { Prevalence of hypertension is } 46 \% \text { in this population. Growth } \\
\text { hormone has antinatriuretic actions which leads to sodium } \\
\text { retention, resulting in volume expansion, increased systolic } \\
\text { output, and increased heart rate (24). }\end{array}$ \\
\hline
\end{tabular}

The Endocrine Society recommends screening for primary hyperaldosteronism in patients who have sustained hypertension resistant to three antihypertensive medications, including a diuretic, or in patients with controlled hypertension on four or more antihypertensive medications (6). Additionally, other patients who should be screened include those with hypertension and hypokalemia, an adrenal incidentaloma, sleep apnea, patients $<40$ years old with a cerebrovascular accident, or patients who have a first degree relative with primary hyperaldosteronism (6). More extensive workup should be done in young adults (age < 30 years old) presenting with resistant hypertension, without risk factors or family history, as they may have hypertension from other causes including renal parenchymal or vascular disease. The purpose of screening is to identify those patients who can potentially benefit from ameliorative or curative surgical and/or medical intervention to significantly reduce cardiovascular morbidity.

\section{PRIMARY HYPERALDOSTERONISM}

Primary hyperaldosteronism is characterized by unregulatedaldosteroneproduction. Themostcommon cause of primary hyperaldosteronism is bilateral adrenal hyperplasia, and the second most common, representing approximately $40 \%$ of cases, are due to aldosterone-producing adrenocortical adenomas. 
Primary Hyperaldosteronism: Screening, Diagnosis, and Management for the Clinician

Other rare etiologies include unilateral adrenal hyperplasia, aldosterone-secreting adrenocortical carcinomas, ectopic aldosterone-producing tumors, and familial hyperaldosteronism (6). Of the secondary causes of hypertension, primary hyperaldosteronism was previously cited as having a prevalence of less than $1 \%$ of patients with hypertension. However, newer studies with cross-sectional and prospective data suggest that this may actually be falsely understated and is present in greater than $10 \%$ of hypertensive patients $(6,3)$. Up to $23 \%$ of patients with resistant hypertension carry a diagnosis of hyperaldosteronism (15).

Aldosterone is a mineralocorticoid that is produced by the zona glomerulosa of the adrenal cortex as an end-product of the renin-angiotensin-aldosterone axis. Aldosterone is created in response to the body's need for sodium retention, which plays a pivotal role for water and sodium homeostasis (5). Aldosterone exerts its action on the principle cells of the late distal convoluted tubules and renal collecting ducts to promote the action of sodium/ potassium-ATPase, leading to increased sodium reabsorption and potassium secretion. Aldosterone secretion is regulated by the renin-angiotensin system (RAS) via stimulation from angiotensin II in response to low perfusion states (figure 1). In primary hyperaldosteronism, aldosterone secretion is increased independently from the RAS, leading to increased sodium reabsorption and thereby increased extracellular fluid volume and blood pressure (18).

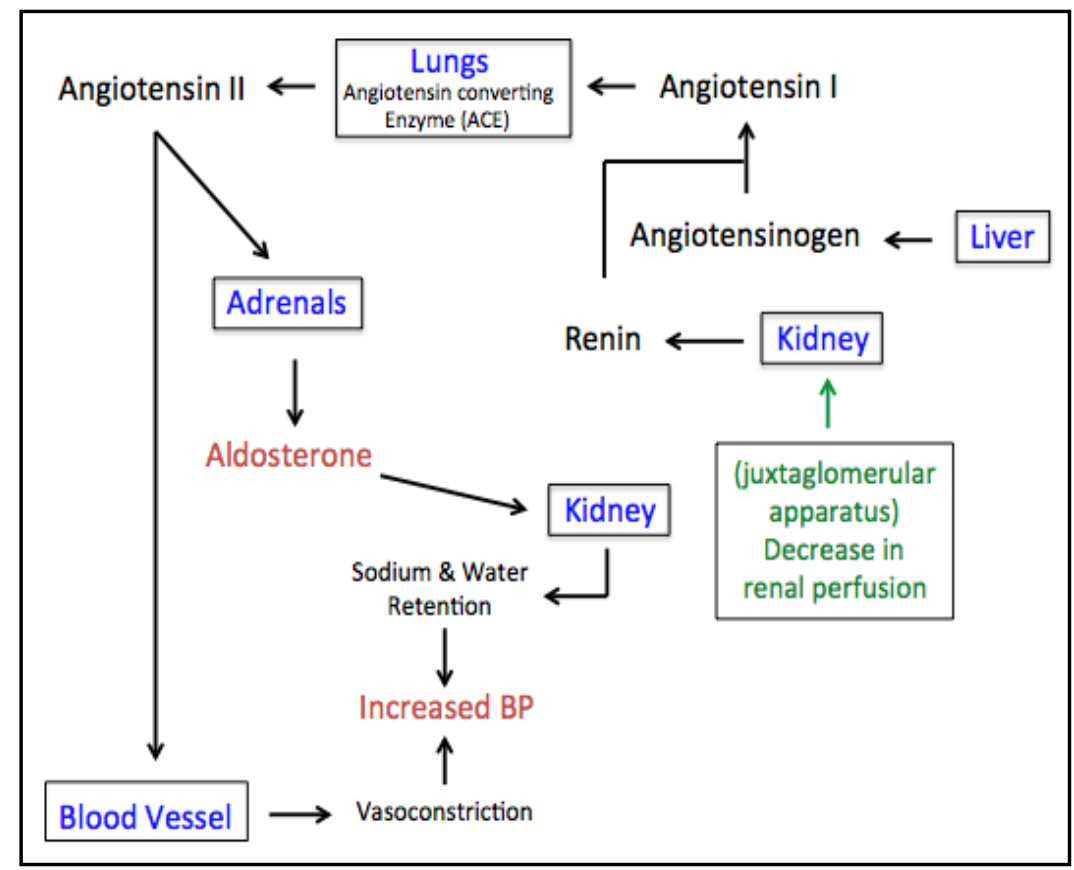

Figure 1. Renin-angiotensin-aldosterone system in normal physiologic state

\section{Clinical Presentation}

Clinical symptoms of primary hyperaldosteronism are nonspecific, though in general patients may report a history of fatigue, muscle weakness, polyuria, polydipsia and constipation. Despite the increase in aldosterone, only a minority of patients with primary hyperaldosteronism have hypokalemia, with a reported prevalence of $9-37 \%$. Therefore, normokalemic hypertension constitutes the most common presentation of the disease, with hypokalemia likely more prevalent in more severe cases (6). Hypokalemia may also be accompanied by metabolic alkalosis, excessive urinary sodium excretion, and hypernatremia. Patients with primary hyperaldosteronism have higher cardiovascular morbidity and mortality than age- and sex-matched patients with essential hypertension and same degree of blood pressure elevation (18). Patients could have symptoms of progressive heart failure and imaging may reveal left ventricular hypertrophy and myocardial fibrosis (15). 


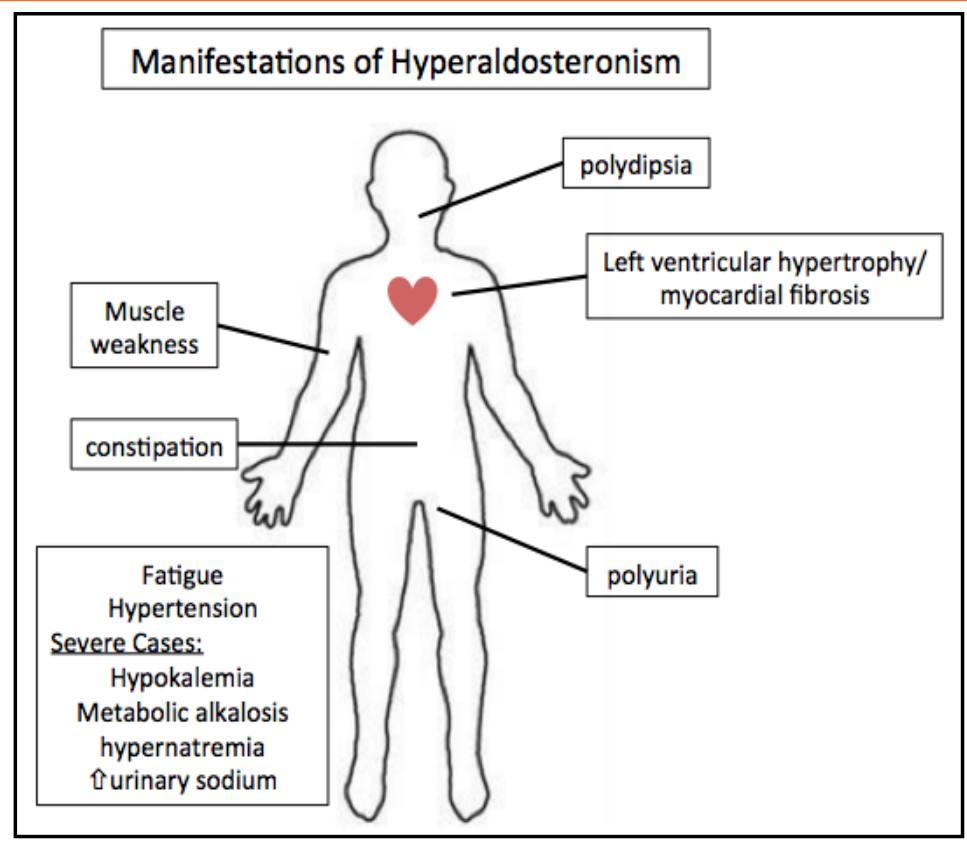

Figure 2. Clinical manifestations of hyperaldosteronism.

\section{SCREENING FOR HYPERALDOSTERONISM}

If there is clinical suspicion that a patient has hyperaldosteronism, the three screening blood tests to order are the plasma aldosterone concentration (PAC), plasma renin activity (PRA), and aldosterone/ renin ratio (PAC/PRA). Currently, the most reliable screening method is the PAC/PRA, also labeled as aldosterone to renin ratio (ARR) according to the Endocrine Society task force. However, various sources have conflicting opinions on the most reliable screening method. Regardless, the consensus is that there needs to be increased aldosterone production and suppressed renin activity in this disease. Thereby, the ratio itself is dependent on these two factors. There are two immunometric assay methods available to measure renin. One method measures the plasma renin activity (PRA, $\mathrm{ng} / \mathrm{mL} / \mathrm{h}$ ), while the other measures the direct renin concentration (DRC, $\mathrm{mU} / \mathrm{L}$ ). A positive test constitutes an aldosterone to renin ratio of $\geqq 30$ when measured using the plasma aldosterone concentration (PAC) to PRA, or a ratio of $\geqq 2.5$ when measured using the PAC to DRC. This ratio is highly dependent on the accuracy of the renin value, which in turn is dependent on the lab. A very low or undetectable denominator (renin value) may give a false positive ratio despite the presence of a low aldosterone concentration. The laboratory performing the assay should have equipment sensitive enough to detect PRA of 0.2-0.3 ng/mL/h (or DRC of $2 \mathrm{mU} / \mathrm{L}$ ).
In addition, the laboratory should report both the individual PAC and the PRA/DRC values in addition to the ARR. It has been suggested that a PAC of $>15 \mathrm{ng} /$ dL should also be included in the criteria in addition to the ARR of $\geqq 30$ (or $\geqq 2.5$ for the case of PAC:DRC) or $>20$ in highly suspicious cases for a positive screening result. However, one study reports PAC values of $<15$ in 16 of 37 patients with confirmed primary hyperaldosteronism, thereby making its inclusion in the diagnostic criteria uncertain (13).

It is important to note that various factors could affect the ARR and therefore can interfere with primary aldosteronism screening. Mineralocorticoid antagonists and diuretics will skew the ARR due to their stronger effect on increasing the plasma renin concentration more than plasma aldosterone, and thereby should also be withdrawn or discontinued for at least 4 weeks prior to testing $(17,18)$. Other medications including central agonists, beta adrenergic blockers, renin inhibitor, ACE inhibitors and ARBs have varying effects on the plasma renin and aldosterone values and will thereby also skew the ARR (table 1) (17). These agents are generally withdrawn for at least 2 weeks and can be replaced with alternative antihypertensive agents during workup of hyperaldosteronism. Antihypertensive agents that can be used which have minimal effects on the ARR include alpha- 1 blockers (prazosin, doxazosin, terazosin), hydralazine, slow-release verapamil, and methyldopa. 
Primary Hyperaldosteronism: Screening, Diagnosis, and Management for the Clinician

The timing of screening is also important as renin secretion has a diurnal variation with the highest levels measured in the morning. The PAC and PRA are typically collected during this time, between 8:00 AM and 10:00 AM. Potassium levels should be replaced if needed since hypokalemia exhibits an inhibitory effect on aldosterone secretion. Patients do not need to be on a salt-restricted diet as hyponatremia may stimulate renin release (17). Once these factors are taken into account and mitigated, a high PAC:PRA ratio constitutes a positive screening test and should be followed by a confirmatory test before subtype classification.

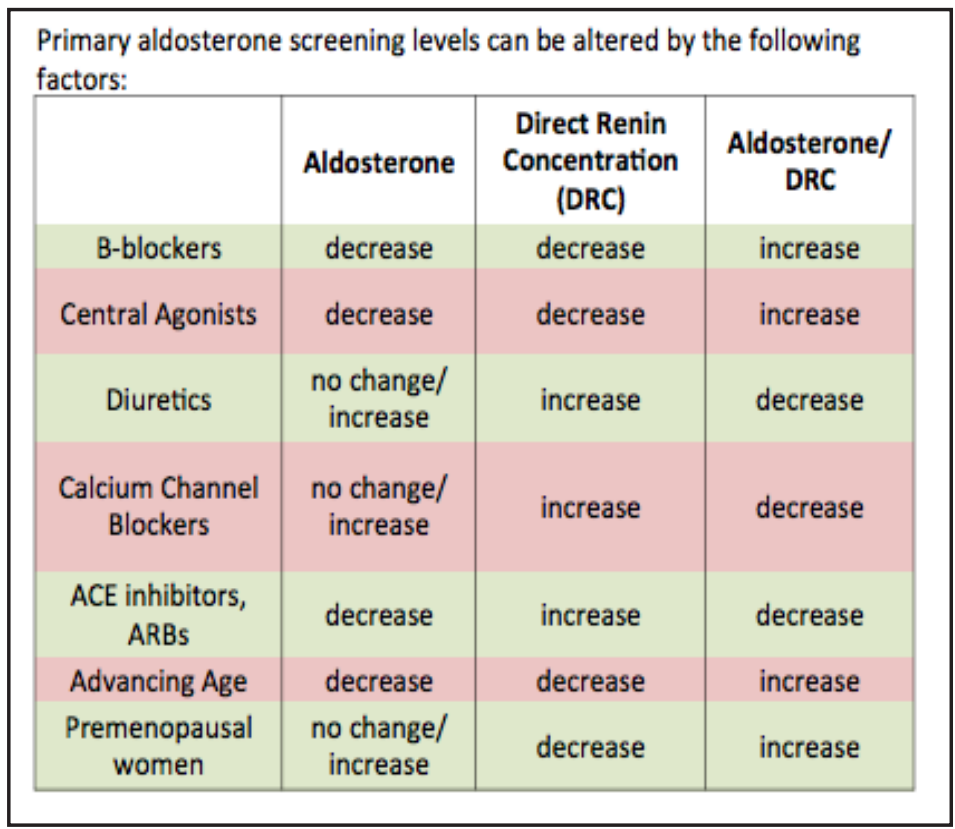

Table 2. Factors that affect aldosterone and renin levels.

\section{Confirmation of THE Diagnosis}

In most patients, an elevated PAC/PRA ratio alone does not establish the diagnosis of primary aldosteronism. The challenge in using ARR is that many factors confound interpretation of the results, including volume status, age, posture, time of day, potassium level, dietary sodium, renal disease, and medications used, as was previously discussed. Moreover, other mineralocorticoid excess states could present with low renin which falsely elevates the ARR ratio. When the patient have a positive ARR screening result, the clinician should order at least one or more confirmatory tests to effectively rule out or rule in the diagnosis of PA by demonstrating inappropriate aldosterone secretion. The exception to the requirement for confirmatory testing is the patient presenting with spontaneous hypokalemia, low PRA, and PAC $>250 \mathrm{pg} / \mathrm{ml}$. In this clinical setting, there is no other diagnosis possible except primary aldosteronism (14). The clinician should proceed with CT scan in these individuals to guide further management, as will later be discussed (18). For the diagnosis of PA, a "gold standard" confirmatory test does not exist, however there are various types of confirmatory tests that are institution-dependent. The four most common methods are the oral salt loading test, intravenous saline infusion test, fludrocortisone suppression test, and captopril test.

\section{Salt Loading Test}

Patients may undergo an oral sodium loading test that involves consuming a high sodium diet over three days, after hypertension is controlled. Patients are usually given guidance on the sodium content in their diet and need to consume $5000 \mathrm{mg}$ of sodium (around 218 $\mathrm{mEq}$ ) per day. Alternatively, patients can be given oral sodium chloride tablets of two $1000 \mathrm{mg}$ to be taken three times a day. Risks of this test include severe hypertension and kaliuresis, hence serum potassium may need to be monitored daily and replaced as indicated. Due to these risks, this test are advised not to be administered with patients with cardiac arrhythmias, severe hypokalemia, uncontrolled hypertension, and renal insufficiency (6). On the third day of the high sodium diet, serum electrolytes and 24-hour urine specimen of aldosterone, creatinine, 
Primary Hyperaldosteronism: Screening, Diagnosis, and Management for the Clinician

and sodium levels are measured. To confirm adequate salt loading urine sodium level should be greater than $200 \mathrm{mEq}$, otherwise results will be inconclusive. Urine aldosterone excretion greater than $12 \mathrm{mcg}$ in $24 \mathrm{hrs}$ is consistent with hyperaldosteronism.

\section{Saline Infusion Test}

The saline infusion test is an alternative test that acts by suppressing endogenous aldosterone production. To perform this test, the patient would be under continuous heart rate and blood pressure monitoring and is initially placed in the recumbent position for one hour. After one hour, 2 liters of intravenous isotonic saline are administered over 4 hours with blood drawn before and after infusion to check serum renin, aldosterone, potassium, and cortisol levels. A post infusion PAC level above $10 \mathrm{ng} / \mathrm{dl}$ is consistent with primary hyperaldosteronism, a PAC below 5-6 $\mathrm{ng} / \mathrm{dl}$ is considered normal, and a PAC of $6-10 \mathrm{ng} / \mathrm{dl}$ is indeterminate. Alternatively, the test can be performed with the patient in a seated position and the post infusion PAC $>6 \mathrm{ng} / \mathrm{dl}$ confirms hyperaldosteronism if the post-infusion cortisol level is less than the initial level (6). Similarly to the oral sodium loading test, due to the high level of sodium intake required for this test patients are at risk for arrhythmias, severe hypokalemia, uncontrolled hypertension, and fluid overload. As such this test should be used with caution in patients with resistant hypertension and congestive heart failure.

\section{Captopril Challenge Test}

To perform this test, the patient begins by staying in an upright position for one hour, either sitting or standing, and then taking oral captopril 25-50 mg. Prior to the test, one hour after, and two hours after the test, the PRA/DRC, plasma aldosterone, and cortisol levels are drawn. Captopril acts by suppressing plasma aldosterone in patients with essential hypertension, but not in most causes of PA thus diagnosis is made by elevated plasma aldosterone levels and decreased renin levels. However, specifically in PA caused by idiopathic adrenal hyperplasia a decrease of plasma aldosterone has been noted. Unfortunately this test has shown a considerable number of equivocal and false negative results $(6,18)$.

\section{Fludrocortisone Suppression Test}

The fludrocortisone suppression test is a less invasive, highly sensitive test for confirming the diagnosis of primary hyperaldosteronism. Fludrocortisone is a mineralocorticoid that promotes sodium reabsorption and potassium secretion. Patients are administered 0.1 mg of fludrocortisone orally every 6 hours for 4 days. Potassium is supplemented with $\mathrm{KCl}$ to keep serum level close to $4.0 \mathrm{mmol} / \mathrm{L}$ and is measured every 6 hours. In addition, supplementation with slow release sodium chloride tablets are used to maintain urinary sodium excretion greater or equal to $3 \mathrm{mmol} / \mathrm{kg}$ body weight. Since the test requires close monitoring, it is recommended that patients be admitted to the hospital, though if a patient is compliant certain outpatient centers can administer the test. On day 4 at $7 \mathrm{AM}$ plasma cortisol is measured and then later at 10 AM while in a seated position, plasma aldosterone, PRA, and plasma cortisol are measured. If the cortisol level at $10 \mathrm{AM}$ is decreased compared to $7 \mathrm{AM}$, then the test can be considered valid as it excludes any possible confounding ACTH effect. A plasma aldosterone of $>6$ ng/dL (170 nmol/L) with PRA $<1 \mathrm{ng} / \mathrm{mL} / \mathrm{h}$ confirms the diagnosis of PA. (6)

\section{Furosemide Upright Test}

The furosemide test is another test suggested to have a $>90 \%$ accuracy in diagnosing PA. Similarly to the previous tests, patients receive $40 \mathrm{mg}$ IV of furosemide and then must stay upright for the duration of the test (26). Labs are drawn twice for PRA/DRC, aldosterone, and potassium, before and two hours after furosemide injection. PRA (plasma renin activity) level $<2$ confirms the diagnosis. Conversely, for essential hypertension, PRA would increase $>2 \mathrm{ng} / \mathrm{ml} / \mathrm{hr}$ after the injection. If the results of the test are inconclusive or equivocal, the next best test is the saline infusion test. Some literature argues that if a patient has no contraindications to the saline infusion test it should be performed instead of the Furosemide test (18).

\section{SubType Classification}

After the diagnosis ofPAisconfirmed, the patientshould undergo further imaging to localize and identify the subtype of PA, to guide further management. Adrenal $\mathrm{CT}$ is recommended as the first line imaging modality to identify abnormalities of the adrenal gland, such as adrenocortical carcinoma or adrenal hyperplasia, due to its cost effectiveness with superior spatial resolution compared to MRI (6). There are limitations to CT as not all APA's are easily detectable unless they 
Primary Hyperaldosteronism: Screening, Diagnosis, and Management for the Clinician

are grossly abnormal in appearance and false positive results correlate with an increase in nonfunctioning adrenal adenomas as the population ages. Due to the unreliability of adrenal CTs, adrenal venous sampling must be done to determine the lateralization of aldosterone production (25). However, adrenal venous sampling can be deferred in the case of a visually apparent adrenocortical carcinoma (6).

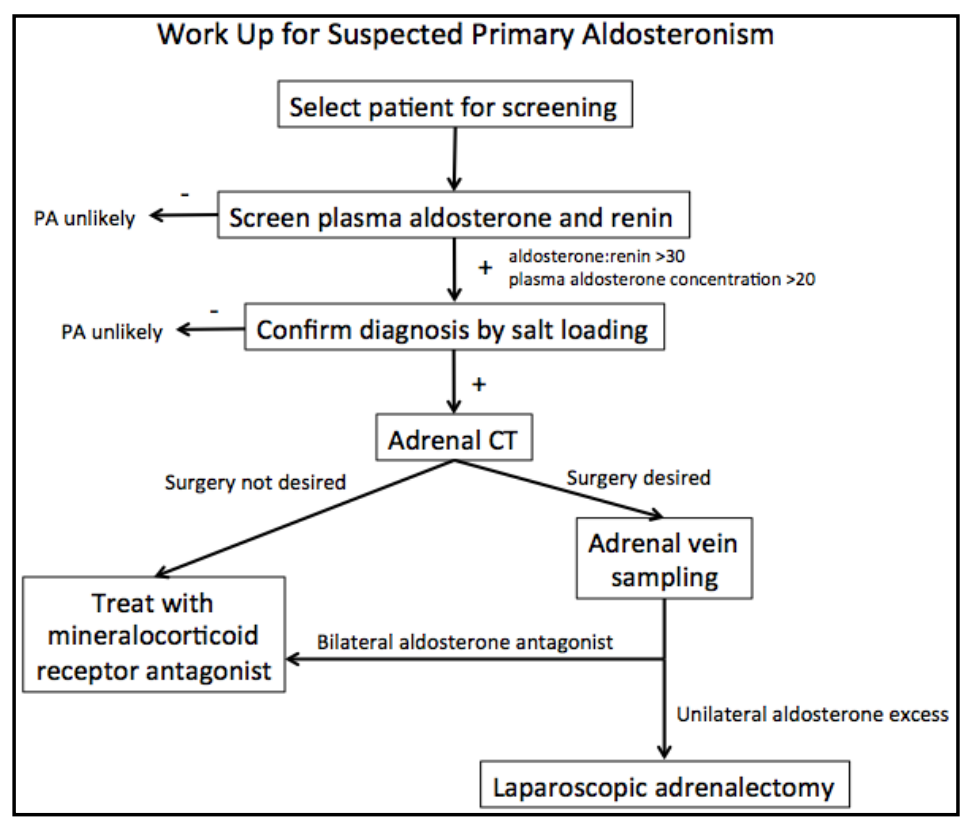

Figure 3. Primary Aldosteronism Work Up Algorithm

Adrenal venous sampling is considered the gold standard diagnostic test, because it determines management by differentiating between unilateral and bilateral adrenal disease. The clinician should determine if a patient is a candidate for surgery prior to adrenal vein sampling due to the complexity and risks, including adrenal hemorrhage, of this invasive procedure. The right angle anatomy of the adrenal vein to the inferior vena cava (IVC) is technically difficult and even experienced radiologists may not be able to cannalize the right adrenal vein. Placement of the catheter in the adrenal vein can be confirmed by a cortisol measurement 10x greater than that in the IVC. During the procedure, the cortisol and aldosterone level in the IVC and bilateral adrenal veins are measured to determine the aldosterone-cortisol ratio (A:C ratio). The A:C ratio will be significantly higher in unilateral disease on the side of the suspected adenoma and the other vein's ratio will be similar to the IVC due to suppression of aldosterone secretion from the zona glomerulosa. Unilateral hypersecretion is confirmed by criteria of a greater than 4-fold difference in $\mathrm{A}: \mathrm{C}$ ratio between the two adrenal veins. If the $\mathrm{A}: \mathrm{C}$ ratio is less than 3 , unilateral hypersecretion is unlikely and an A:C ratio of 3-4 is indeterminate. To minimize aldosterone fluctuations and enhance the cortisol gradient of adrenal vein to IVC, cosyntropin, as a bolus or continuous infusion, may be used to acutely stimulate aldosterone release (6).

One population of patients that can forgo adrenal venous sampling and proceed directly to surgery include younger individuals $<35$ years of age who have high suspicion of unilateral disease and adrenal cortical adenoma on imaging. The target for surgery is apparent in this clinical setting and therefore additional testing before surgery is unnecessary (1).

Table 3. Example of plasma aldosterone (A) and cortisol (C) concentrations from adrenal veins and inferior vena cava.

\begin{tabular}{|c|c|c|c|c|c|c|}
\hline Time & Left, $\mathbf{A}$ & Left, C & Right, A & Left, C & IVC, A & IVC, C \\
\hline $\mathbf{- 5}$ & 2342 & 313 & 125 & 404 & 61 & 19 \\
\hline $\mathbf{0}$ & 2012 & 225 & 120 & 350 & 63 & 21 \\
\hline $\mathbf{2 0}$ & 1818 & 282 & 171 & 231 & 112 & 32 \\
\hline $\mathbf{4 0}$ & 8102 & 361 & 191 & 357 & 225 & 112 \\
\hline
\end{tabular}


Primary Hyperaldosteronism: Screening, Diagnosis, and Management for the Clinician

Values at time -5 and 0 min are baseline values in absence of ACTH stimulation. Values were obtained 20 and 40 minutes after bolus administration of Cosyntropin 250 ug intravenously. The catheter

was positioned correctly in both adrenal veins as demonstrated by greater than 10 -fold increase in concentrations compared to those in the IVC. Aldosterone ng/dL; cortisol ug/dL.

Table 4. Aldosterone:cortisol ratios calculated from examples in table 3.

\begin{tabular}{|c|c|c|c|}
\hline Time & Left, A:C & Right, A:C & IVC, A:C \\
\hline $\mathbf{- 5}$ & 7.5 & 0.3 & 3.2 \\
\hline $\mathbf{0}$ & 8.9 & 0.3 & 3.0 \\
\hline $\mathbf{2 0}$ & 6.4 & 0.7 & 3.5 \\
\hline $\mathbf{4 0}$ & 22.4 & 0.5 & 2.0 \\
\hline
\end{tabular}

Diagnosis of left APA can be made because ratios on the left adrenal vein were greater than 4 -fold in comparison to the contralateral side

\section{TREATMENT}

The overall treatment goal in patients with primary hyperaldosteronism is to prevent adverse outcomes associated with hypertension and hypokalemia. Surgery, if indicated, is the preferred management approach because it corrects the underlying cause, as well as reducing long term expenses for the patient (7).

Surgical candidates include patients who are confirmed to have unilateral aldosterone-producing adenoma or unilateral adrenal hyperplasia, that have been diagnosed and subtyped by CT imaging and adrenal venous sampling. The recommended surgery for treatment is unilateral laparoscopic adrenalectomy, as it has been shown to correct hypokalemia and even cure hypertension in 30-60\% of patients (6). Prior to surgery, the patient should be treated with a mineralocorticoid antagonist such as spironolactone or eplerenone to normalize potassium levels. Pretreatment decreases the risk of hyporeninemic hypoaldosteronism in the immediate postoperative period by increasing and normalizing serum renin levels through inhibition of aldosterone.

Patients with bilateral adrenal disease and those who are not surgical candidates should be managed medically. Lifestyle and risk versus benefits must be considered prior to initiation of medications as these patients frequently require multiple antihypertensive agents, increasing the cost and polypharmacy side effects.

Regardless, mineralocorticoid-receptor antagonists remain a mainstay of treatment with spironolactone as the first choice given its superiorblood pressure control effects. Initial dosing is $25 \mathrm{mg}$ daily with titration up to maximum of $200 \mathrm{mg}$ twice a day. Common side effects include painful gynecomastia, erectile dysfunction, decreased libido in men, and menstrual irregularity in women due to cross reactivity with sex steroid receptors (27). In contrast, eplerenone is a selective mineralocorticoid receptor antagonist, minimizing side effects but with decreased efficacy on blood pressure. Dosing starts at $25 \mathrm{mg}$ twice daily and could be titrated up to max of $100 \mathrm{mg}$.

Amiloride is a potassium-sparing diuretic that is often used as the drug of choice for patients who could not tolerate spironolactone and eplerenone. It is equally as effective in controlling blood pressure and has more benign side effects than spironolactone. However, side effects of gynecomastia and impotence are unchanged (21). Aldosterone synthase inhibitors (ASI) which directly antagonise the production of aldosterone, are currently being researched and may be a future treatment option. A few ASI under research include BI689648, FAD286, LCI699. Although the proposed mechanism of ASI is promising for the reversal of the plethora of deleterious side effects due to unregulated aldosterone production, its successful development has been difficult due to the similarity between aldosterone synthase and cortisol synthase (19). Additionally, despite studies showing normalization of potassium levels in patients treated with ASI, hypertension and sodium levels have been unaffected. Further investigation would be needed before it becomes a viable option over mineralocorticoid antagonists (20).

\section{CoNCLUSION}

Primary hyperaldosteronism may be more common among patients with hypertension than historically estimated. Screening in patients with resistant hypertension should include measurement of ARR and a positive finding should be verified with one 
Primary Hyperaldosteronism: Screening, Diagnosis, and Management for the Clinician

of the confirmatory tests, such as salt loading test, saline infusion test, furosemide upright test, captopril challenge test, or fludrocortisone suppression test. Once confirmed, adrenal imaging is done to provide a morphologic diagnosis. Surgery is generally recommended in patients with aldosterone-producing adenomas but this must be confirmed with adrenal venous sampling to differentiate from bilateral disease which is only amenable to medical treatment. Should medical treatment be sought, patients are typically treated with mineralocorticoid antagonists in adjunct with other antihypertensive agents to control hypertension. The goal of either treatment, whether medical or surgical, is to improve blood pressure and correct electrolyte abnormalities in order to address risks of cardiovascular and renal morbidities.

\section{REFERENCES}

[1] Amar L, Azizi M, Menard J, Peyrard S, Plouin PF. Sequential Comparison of Aldosterone Synthase Inhibition and Mineralocorticoid Blockade in Patients With Primary Aldosteronism. J Hypertens. 2013. 31(3):624-9.

[2] Biglieri EG, Herron MA, Brust N. 17-Hydroxylation Deficiency in Man. J Clin Invest. 1966; 45:1946.

[3] Douma S, Petidis K, Doumas M, Papefthimiou P, et al. Prevalence of Primary Hyperaldosteronism in Resistant Hypertension: A Retrospective Observational Study. Lancet. 2008; 371 (9628):1921.

[4] Fryar CD, Ostchega Y, Hales CM, Zhang G, KruszonMoran D. Hypertension Prevalence and Control Among Adults: United States, 2015-2016. NCHS data brief, no 289. Hyattsville, MD: National Center for Health Statistics. 2017.

[5] Fuller PJ, Young MJ. Mechanisms of mineralocorticoid action. Hypertension. 2005; 46:1227.

[6] Funder JW, Carey RM, Mantero F, et al. The Management of Primary Aldosteronism: Case Detection, Diagnosis, and Treatment: An Endocrine Society Clinical Practice Guide. J Clin Endocrinol Metab. 2016; 101:1889-1916.

[7] Galati, SJ. Primary Aldosteronism Challenges in Diagnosis and Management. Endocrinol Metab Clin North Am. 2015; 355-369.

[8] Iglesias P, Acosta M, Sánchez R, Fernández-Reyes
MJ, Mon C, Díez JJ. Ambulatory Blood Pressure Monitoring in Patients with Hyperthyroidism Before and After Control of Thyroid Function. Clin Endocrinol. 2005; 63:66-72. (Abstract only)

[9] Lind L, Ljunghall S. Pre-operative Evaluation of Risk Factors for Complications in Patients With Primary Hyperparathyroidism. Eur J Clin Invest. 1995; 25(12):955.

[10] Logan AG, Perlikowski SM, Mente A, Tisler A, Tkacover R, Niroumand M, Leung RS, Bradley TD. High Prevalence of Unrecognized Sleep Apnoea in Drug-Resistant Hypertension. J Hypertens. 2001 Dec; 19(12):2271-7.

[11] Merai R, Siegel C, Rakotz M, Basch P, Wright J, Wong B; DHSc., Thorpe P. CDC Grand Rounds: A Public Health Approach to Detect and Control Hypertension. MMWR Morb Mortal Wkly Rep. 2016 Nov 18; 65(45):1261-1264.

[12] Milliez P, Girerd X, Plouin PF, Blacher J, Safar ME, Mourad JJ. Evidence for an Increased Rate of Cardiovascular Events in Patients with Primary Aldosteronism. J Am Coll Cardiol. 2005; 45:12431248.

[13] Mosso L, Carvajal C, Gonzalez A, et al. Primary Aldosteronism and Hypertensive Disease. Hypertension. 2003; 42:161-165.

[14] Nanba K, Tamanaha T, Nakao K, Kawashima ST, Usui T, Tagami T, et al. Confirmatory Testing in Primary Aldosteronism. J Clin Endocrinol Metab. 2012; 97(5):1688-94.

[15] Rimoldi SF, Scherrer U, Messerli FH. Secondary Arterial Hypertension: When, Who, and How to Screen? Eur Heart J. 2014; 35:1245-1254.

[16] Speiser PW, White PC. Congenital Adrenal Hyperplasia. N Engl J Med. 2003; 349:776.

[17] Stowasser M, Gordon R, Rutherford J, Nikwan N, Daunt N, Slater G. Diagnosis and Management of Primary Aldosteronism. J Renin Angiotensin Aldosterone Syst. 2001 Dec; 2(3):156-169.

[18] Vilela LAP, Almeida MQ. Diagnosis and Management of Primary Aldosteronism. Arch Endocrinol Metab. 2017; 61/3.

[19] Weldon S, Cerny M, et al. Selectivity of BI 689648, a Novel, Highly Selective Aldosterone Synthase Inhibitor: Comparison with FAD286and LCI699 in 
Primary Hyperaldosteronism: Screening, Diagnosis, and Management for the Clinician

Nonhuman Primates. J Pharm and Experimental Therapeutics. 2016 Oct; 359(1):142-150.

[20] Amar L, Azizi M, et al. Aldosterone Synthase Inhibition with LCI699 A Proof-of-Concept Study in Patients With Primary Aldosteronism. J Hypertension. 2010; 56(5):831-838.

[21] Macfie HL, Colvin CL, Anderson PO. New drug evaluations amiloride (Midamor, Merck, Sharp and Dohme). Drug Intell Clin Pharm. 1981;15(2):94-98. [PubMed 7274028]

[22] Dernellis J, Panaretou M. Effects of thyroid replacement therapy on arterial blood pressure in patients with hypertension and hypothyroidism. Am Heart J. April 2002;143:718-24.

[23] HelmutSchifflandSusanneM.Lang, "Hypertension Secondary to PHPT: Cause or Coincidence?," International Journal of Endocrinology, vol. 2011, Article ID 974647, 6 pages, 2011. https:// doi.org/10.1155/2011/974647.

[24] Vitale, Giovanni, et al. "Hypertension in Acromegaly and in the Normal Population: Prevalence and Determinants." Clinical Endocrinology, vol. 63, no. 4, Oct. 2005, pp. 470476., doi:10.1111/j.1365-2265.2005.02370.x.
[25] Magill SB, Raff H, Shaker JL, et al. Comparison of Adrenal Vein Sampling and Computed Tomography in the Differentiation of Primary Aldosteronism. J Clin Endocrinol Metab. 2001; 86:1066-1071.

[26] Kaplan NM, Kem DC, Holland OB, Kramer NJ, Higgins J, Gomez-Sanchez C. The Intravenous Furosemide Test: A Simple Way to Evaluate Renin Responsiveness. Ann Intern Med. 1976;84:639645. doi: 10.7326/0003-4819-84-6-639

[27] Aronova, Anna, Thomas J Fahey III, and Rasa Zarnegar. "Management of Hypertension in Primary Aldosteronism." World Journal of Cardiology 6.5 (2014): 227-233. PMC. Web. 20 Aug. 2018.

[28] Ozcelik, Serhat, et al. "Relationship of parathyroid adenoma volume with preoperative biochemical parameters." Age (years)53.14.996 (2018): 19-84

[29] Celik, Mehmet, et al. "Benign and malignant thyroid gland disease in the patients with primary hyperparathyroidism." International Journal of Applied and Basic Medical Research 7.2 (2017): 117.

Citation: Winnie Nhan, DO, Quang T. Nguyen, DO, FACP, FACE, FTOS. et al. Primary Hyperaldosteronism: Screening, Diagnosis, and Management for the Clinician. Archives of Diabetes and Endocrine System. 2018; 1(2): 39-48.

Copyright: (C) 2018 Winnie Nhan, DO, Quang T. Nguyen, DO, FACP, FACE, FTOS. et al. This is an open access article distributed under the Creative Commons Attribution License, which permits unrestricted use, distribution, and reproduction in any medium, provided the original work is properly cited. 